\title{
Performance of MEGARA Spectrograph optical elements
}

\author{
E. Carrasco*a , G. Páez ${ }^{\mathrm{b}}$, R. Izazaga ${ }^{\mathrm{a}}$, J. de la Luz Hurtado ${ }^{\mathrm{b}}$, C. Pérez ${ }^{\mathrm{b}}$, F. Granados ${ }^{\mathrm{a}}$, D. Aguirre ${ }^{\mathrm{c}}$, E. \\ Percino $^{\text {a }}$ J. Reyes ${ }^{a}$, A. Gil de Paz ${ }^{\mathrm{d}}$, J. Gallego ${ }^{\mathrm{d}} \&$ J. Iglesias ${ }^{\mathrm{e}}$ \\ ${ }^{a}$ Instituto Nacional de Astrofísica, Óptica y Electrónica (INAOE, Mexico); ${ }^{b}$ Centro de \\ Investigaciones en Óptica (CIO, Mexico); 'universidad Autónoma de México, (UNAM, México); \\ ${ }^{\mathrm{d}}$ Universidad Complutense de Madrid (UCM, Spain); ${ }^{\mathrm{e}}$ Instituto de Astrofísica de Andalucía-CSIC
}

(IAA. Spain)

\begin{abstract}
MEGARA is the new IFU and multiobject spectrograph for Gran Telescopio Canarias. The spectograph will offer spectral resolution $\mathrm{R}_{\mathrm{fwhm}} \sim 6,000,12,000$ and 18,700. Except for the optical fibers and microlenses, the complete MEGARA optical system has been manufactured in Mexico. This includes a field lens, a 5-lenses collimator, a 7-lenses camera and a complete set of volume phase holographic gratings with 36 flat windows and 24 prisms. All these elements are very large and complex, with very efficient antireflection coatings. Here the optical performance of MEGARA collimator and camera lenses and the field lens is presented.
\end{abstract}

Keywords: Spectrographs, high precision optics, optical testing, coatings

\section{INTRODUCTION}

An overview of the science and the instrument status is described by Gil de Paz, A. et al. [1]; The detector cryogenic system by Ferrusca, D., et al. [2]; The fiber MOS positioning tool by Iglesias-Páramo, J., et al. [3]; MEGARA fiber MOS assignment tool (FMAT) by Gómez-Alvarez. P., et al. [4]; MEGARA exposure time calculator by Castillo-Morale, A., et al. [5]; MEGARA control system by Lefort, B., et al. [6]; MEGARA spectrograph mechanics and opto-mechanics in the AIV phase by Maldonado.M., et al. [7]; The large MEGARA pupil elements: assembly, tests, and performance by Martínez-Delgado, I., et al. [8]; MEGARA: high-precision alignment system for gluing fibers and microlenses by PérezCalpena. A., et al. [9]. In this paper we focus in the spectrograph main optics. The spectrograph optics is formed by 5lenses, one of them aspheric and 7-lenses camera. The polishing techniques for MEGARA optics are presented by IzazagaPérez, R., et al. [10]. Except for the aspheric all of them were manufactured at INAOE and CIO. The coatings evaporations were carried out at CIO, the results are presented by Ortiz. R., et al. [11]. Here we described he optical performance of MEGARA main optics and field lens.

\subsection{Collimator and camera optics}

The $\mathrm{f} / 3$ collimator has a focal length of $484.4 \mathrm{~mm}$ for a singlet and two doblets. The $\mathrm{f} / 1.5$ camera has a focal length of $245.9 \mathrm{~mm}$ for two doublets and three singlets. The last lens (CAM-S7) is also the cryostat window. In Table 1 a summary of MEGARA lenses specifications is presented. Given the diameters and specifications it is considered high precision optics. Except for the OLL-S1, manufactured by HOYA, all the lenses were manufactured at INAOE and CIO.

*bec@inaoep.mx; phone 52222 2663100; inaoep.mx

Ground-based and Airborne Instrumentation for Astronomy VI, edited by Christopher J. Evans, Luc Simard, Hideki Takami Proc. of SPIE Vol. 9908, 990885 · C 2016 SPIE · CCC code: 0277-786X/16/\$18 · doi: 10.1117/12.2232208 
Table 1. MEGARA lenses specifications summary.

\begin{tabular}{|c|c|c|c|c|c|c|c|c|}
\hline Element & Material & $\begin{array}{c}\text { Edge } \varnothing \\
(\mathrm{mm}) \\
-0.015\end{array}$ & $\begin{array}{c}\text { Thickness } \\
\text { (mm) }\end{array}$ & $\begin{array}{c}\begin{array}{c}\mathrm{RoC} \\
(\mathrm{mm})\end{array} \\
\mathrm{S} 1\end{array}$ & $\begin{array}{c}\mathrm{RoC} \\
(\mathrm{mm}) \\
\mathrm{S} 2\end{array}$ & $\begin{array}{c}\begin{array}{c}\text { Fringes @ } \\
\lambda=0.632 \mathrm{~nm} \\
\mathrm{~S} 1 / \mathrm{S} 2\end{array}\end{array}$ & $\begin{array}{c}\text { S/D } \\
\text { S1-S2 }\end{array}$ & $\begin{array}{l}\text { Wedge } \\
\text { (arcmi } \\
\text { n) }\end{array}$ \\
\hline Field & F. Silica & 274 & $30.0 \pm 0.1$ & $-214.6 \pm 2$ & $-1731.8 \pm 2$ & $0.25 / 0.25$ & $20 / 10-20 / 10$ & 2 \\
\hline COLL-S1 & PBM2Y & 155 & $35.0 \pm 0.15$ & $-97 \pm 0.1$ & $-113.3 \pm 0.1$ & $0.25 / 0.25$ & $40 / 20-20 / 10$ & 2 \\
\hline COLL-D2 & PBM2Y & 272 & $35.0 \pm 0.15$ & FLAT & $-728.1 \pm 1$ & $0.5 / 1$ & $20 / 10-40 / 20$ & 2 \\
\hline COLL-D3 & BSM51Y & 272 & $35.0 \pm 0.15$ & $-728.1 \pm 1$ & $-398.8 \pm 0.4$ & $1 / 0.5$ & $20 / 10-40 / 20$ & 2 \\
\hline COLL-D4 & PBM8Y & 260 & $48.5 \pm 0.15$ & $+1259.9 \pm 2$ & $+344.5 \pm 0.5$ & $0.5 / 1$ & $40 / 20-20 / 10$ & 2 \\
\hline COLL-D5 & $\mathrm{CaF} 2$ & 250 & $45.0 \pm 0.15$ & $+344.5 \pm 0.5$ & $-542.5 \pm 0.5$ & $0.5 / 2$ & $60 / 40-40 / 20$ & 2 \\
\hline CAM-D1 & $\mathrm{CaF} 2$ & 236 & $60.0 \pm 0.10$ & $+435.9 \pm 0.4$ & $-231.7 \pm 0.2$ & $0.5 / 1$ & $40 / 20-60 / 40$ & 2 \\
\hline CAM-D2 & BSM51Y & 240 & $25.0 \pm 0.1$ & $-231.7 \pm 0.2$ & FLAT & $1 / 0.5$ & $40 / 20-20 / 10$ & 2 \\
\hline CAM-D3 & BAL15Y & 240 & $25.0 \pm 0.1$ & $+269.2 \pm 0.2$ & $+145.1 \pm 0.1$ & $0.5 / 1$ & $20 / 10-40 / 20$ & 2 \\
\hline CAM-D4 & $\mathrm{CaF} 2$ & 220 & $60.0 \pm 0.1$ & $+145.1 \pm 0.1$ & FLAT & $1 / 0.5$ & $40 / 20-40 / 20$ & 2 \\
\hline CAM-S5 & $\mathrm{CaF} 2$ & 220 & $62.0 \pm 0.1$ & $+156 \pm 0.1$ & $-1143 \pm 0.8$ & $0.5 / / 0.5$ & $40 / 20-40 / 20$ & 2 \\
\hline CAM-S6 & S-LAH55 & 140 & $40.0 \pm 0.1$ & $+176.4 \pm 0.2$ & $+365.8 \pm 0.3$ & $0.5 / 0.5$ & $20 / 10-20 / 10$ & 2 \\
\hline CAM-S7 & S-NBH8 & 110 & $30.0 \pm 0.1$ & $-162.5 \pm 0.2$ & $+219.5 \pm 0.2$ & $0.25 / 0.25$ & $20 / 10-20 / 10$ & 2 \\
\hline
\end{tabular}

\subsection{Results}

The following tables we present the as-built parameters of the individual elements manufactured at INAOE and CIO. When a given specifation was not achieved, the system optical performance was analysed by the optical designer and when accepted a Non Conformity (N/C) was recorded. 
Table 2. Collimator lens COLL-D2 as-built parameters.

\begin{tabular}{|c|c|c|c|c|c|}
\hline \begin{tabular}{|c|} 
AIV-TEC-MEG-084- \\
3.3 \\
\end{tabular} & & Manufacturer & INAOE & & \\
\hline \multirow{2}{*}{\multicolumn{6}{|c|}{$\begin{array}{c}\text { COLL-D2 } \\
\text { Ohara PBM2Y }\end{array}$}} \\
\hline & & & & & \\
\hline Parameter & Requirement & Measurement & Measurement & Measurement & Instrument or method \\
\hline \begin{tabular}{|l|} 
Surface 1 \\
\end{tabular} & & Test plate & Surface 1 & & \\
\hline $\operatorname{RoC}(\mathrm{mm})$ & Flat & & & & $\begin{array}{c}\text { Interferometer+reference } \\
\text { flat }\end{array}$ \\
\hline Used aperture $\Phi(\mathrm{mm})$ & $250 \times 100$ & - & $\Phi=250$ & - & \\
\hline Irr in used aperture & 2 fr & - & 0.8134 & - & ZYGO Interferometer \\
\hline Irr in $\Phi=90$ & $0.5 \mathrm{fr}$ & - & 0.0614 & - & ZYGO Interferometer \\
\hline MII 13830 SD & $20 / 10$ & - & $20 / 10$ & - & $\begin{array}{l}\text { Visual inspection vs. } \\
\text { calibrated reference }\end{array}$ \\
\hline Protective chamfer & $1.0 \times 45^{\circ}$ & - & $1.0 \times 45^{\circ}$ & - & Graduated reticle \\
\hline \multicolumn{6}{|c|}{\begin{tabular}{|l|l|} 
AR Coating 370-980 & \\
mm & \\
$R>1.3 \%$ & Tav $>98.7 \%$ \\
\end{tabular}} \\
\hline Surface 2 & & & Surface 2 & Surface2 & \\
\hline RoC (mm) & $-728.1+/-1$ & -728.1 & & & Nodal slide optical bench \\
\hline Used aperture $\Phi(\mathrm{mm})$ & $250 \times 120$ & & & & \\
\hline Ir in used aperture & $3 \mathrm{fr}$ & $\begin{array}{c}0.3385 \mathrm{in} \\
\Phi=140 \mathrm{~mm}\end{array}$ & & 0.3113 & $\begin{array}{c}\text { Newton interf./ } \\
\text { subapertures stitching }\end{array}$ \\
\hline Irr in $\Phi=105$ & $1 \mathrm{fr}$ & 0.1887 & & 0.1221 & $\begin{array}{l}\text { Newton } \\
\text { interf./subapertures } \\
\text { stitching }\end{array}$ \\
\hline MII 13830 SD & $40 / 20$ & & $40 / 20$ & & $\begin{array}{l}\text { Visual inspection vs. } \\
\text { calibrated reference }\end{array}$ \\
\hline Protective chamfer & $1.0 \times 45^{\circ}$ & _ & $1.0 \times 45^{\circ}$ & - & Graduated reticle \\
\hline $\begin{array}{l}\text { AR coating } 370-980 \\
\mathrm{~nm}\end{array}$ & no coating & - & - & - & \\
\hline \multicolumn{6}{|l|}{ Lens } \\
\hline Edge diameter (mm) & $272+0 /-0.015$ & & $272.1(* \star)^{1}$ & & Mitutoyo $\mathrm{cal} \pm 0.010$ \\
\hline $\begin{array}{l}\text { Central thickness* } \\
(\mathrm{mm})\end{array}$ & $35+/-0.1$ & & 34.96 & & Mitutoyo cal \pm 0.010 \\
\hline Wedge (arcmin) & 2 & & 0.02 & & Mitutoyo cal \pm 0.010 \\
\hline
\end{tabular}

Proc. of SPIE Vol. 9908 990885-3 
Table 3. Collimator lens COLL-D3 as-built parameters.

\begin{tabular}{|c|c|c|c|c|c|}
\hline AIV-TEC-MEG-084-3.4 & & Manufacturer & INAOE & & \\
\hline \multicolumn{6}{|l|}{ COLL-D3 } \\
\hline \multicolumn{6}{|l|}{ Ohara BSM51Y } \\
\hline Parameter & Requirement & Measurement & Measurement & Measurement & Instrument or method \\
\hline Surface 1 & & Test plate & Surface 1 & & \\
\hline $\operatorname{RoC}(\mathrm{mm})$ & $-728.1+/-1$ & - & -728.1 & - & Nodal slide optical bench \\
\hline Used aperture $\Phi(\mathrm{mm})$ & $250 \times 120$ & - & & - & \\
\hline Irr in used aperture & $3 \mathrm{fr}$ & - & 0.268 & - & ZYGO interferometer \\
\hline Irr in $\Phi=105$ & $1 \mathrm{fr}$ & - & 0.095 & - & ZYGO interferometer \\
\hline MIL 13830 SD & $40 / 20$ & - & $30 / 10$ & - & $\begin{array}{l}\text { Visual inspection vs. graduated } \\
\text { reference }\end{array}$ \\
\hline Protective chamfer & $1.0 \times 45^{\circ}$ & - & $1.0 \times 45^{\circ}$ & - & Graduated reticle \\
\hline AR coating $370-980 \mathrm{~nm}$ & no coating & & & & \\
\hline Surface 2 & & & Surface 2 & & \\
\hline $\operatorname{RoC}(\mathrm{mm})$ & $-398.8+-0.4$ & -398.8 & & -398.8 & Nodal slide optical bench \\
\hline Used aperture $\Phi(\mathrm{mm})$ & $250 \times 120$ & & & & \\
\hline Irr in used aperture & $2 \mathrm{fr}$ & $\begin{array}{c}0.3253 \text { in } \\
\Phi=150\end{array}$ & & 0.6715 & $\begin{array}{l}\text { Newton interferometry/ } \\
\text { subapertures stitching }\end{array}$ \\
\hline Irr in $\Phi=105$ & $0.5 \mathrm{fr}$ & 0.1772 & & 0.2057 & $\begin{array}{l}\text { Newton interferometry// } \\
\text { subapertures stitching }\end{array}$ \\
\hline MIL 13830 SD & $20 / 10$ & & $20 / 5$ & & $\begin{array}{l}\text { Visual inspection } v s . \\
\text { graduated reference }\end{array}$ \\
\hline Protective chamfer & $1.0 \times 45^{\circ}$ & & $1.0 \times 45^{\circ}$ & & Graduated reticle \\
\hline $\begin{array}{l}\text { AR Coating } 370-980 \mathrm{~mm} \\
\text { Mean AOI }=8.5^{\circ} ; R<1.3 \%\end{array}$ & Tav $>98.7 \%$ & & Tav $=99.4 \%$ & & CARY spectrometer \\
\hline \multicolumn{6}{|l|}{ Lens } \\
\hline Edge diameter (mm) & $\begin{array}{l}272+0 /- \\
0.015 \\
\end{array}$ & & 271.99 & & Mitutoyo calibrator $=0.010$ \\
\hline Central thickness (mm) & $35+1-0.15$ & & 34.9 & & Mitutoyo calibrator $=0.010$ \\
\hline Wedge (arcmin) & 2 arcmin & & 1.16 & & Centering machine \\
\hline
\end{tabular}


Table 4. Collimator lens COLL-D4 as-built parameters.

\begin{tabular}{|c|c|c|c|c|}
\hline AIV-TEC-MEG-084-3.5 & & Manufacturer & ClO & \\
\hline \multicolumn{5}{|l|}{ COLL-D4 } \\
\hline \multicolumn{5}{|l|}{ Ohara PBM8Y } \\
\hline \multirow[t]{2}{*}{ Parameter } & Requirement & Measurement & Measurement & Instrument or \\
\hline & & & & method \\
\hline Surface 1 & & Test plate & Surface $1 /$ Newton & \\
\hline RoC & $1259.9+/-2 \mathrm{~mm}$ & 1260.8 & 1260.8 & Interferometer \\
\hline Used aperture $\Phi$ & $240 \mathrm{~mm}$ & & & \\
\hline Irr in used aperture & $2 \mathrm{fr}$ & $0.36 \mathrm{fr}$ & $1.14 \mathrm{fr}$ & Interferometer \\
\hline Irr in $\Phi=150$ & $0.5 \mathrm{fr}$ & $\begin{array}{c}0.167 \mathrm{fr} \text { in } \\
\Phi=138\end{array}$ & \multirow{2}{*}{$\begin{array}{l}0.46 \mathrm{fr} \\
20 / 10\end{array}$} & Interferometer \\
\hline MIL 13830 SD & $20 / 10$ & - & & Visual inspection \\
\hline Rms surface roughness & $20 \AA$ & - & & \\
\hline Protective chamfer & $1.0 \times 45^{\circ}$ & - & $1 \times 45^{\circ}$ & Graduated reticule \\
\hline $\begin{array}{l}\text { AR Coating } 370-980 \mathrm{~nm} \\
\text { Mean } \mathrm{AOI}=4.5^{\circ} ; \mathrm{R}<1.3 \%\end{array}$ & Tav $>98.7 \%$ & 一 & Tav $=99.05 \%$ & \\
\hline Surface 2 & & & Surface 2 & \\
\hline RoC & $344.5+/-0.5 \mathrm{~mm}$ & - & 344.53 & Interferometer \\
\hline Used aperture $\Phi$ & $240 \mathrm{~mm}$ & - & & \\
\hline Irr in used aperture & $3 \mathrm{fr}$ & - & $1.6 \mathrm{fr}$ & Interferometer \\
\hline Irr in $\Phi=160$ & $1 \mathrm{fr}$ & - & $0.69 \mathrm{fr}$ & Interferometer \\
\hline MIL 13830 SD & $40 / 20$ & - & $40 / 20$ & Visual inspection \\
\hline Rms surface roughness & $30 \AA$ & - & & \\
\hline Protective chamfer & $1.0 \times 45^{\circ}$ & - & $1.0 \times 45^{\circ}$ & Graduated reticule \\
\hline Flat chamfer & $5 \mathrm{~mm}$ & - & $5.1 \mathrm{~mm}$ & Graduated reticule \\
\hline AR coating $370-980 \mathrm{~nm}$ & No coating & - & - & \\
\hline \multicolumn{5}{|l|}{ Lens } \\
\hline Edge diameter & $260+0 /-0.015$ & - & 259.989 & CMM \\
\hline Central thickness & $25+/-0.15$ & - & 24.889 & CMM \\
\hline Wedge & 2 arcmin & - & 1 arcmin & Centering machine \\
\hline
\end{tabular}

Proc. of SPIE Vol. $9908990885-5$

Downloaded From: http://proceedings.spiedigitallibrary.org/ on 03/08/2017 Terms of Use: http://spiedigitallibrary.org/ss/termsofuse.aspx 
Table 5. Collimator lens COLL-D5 as-built parameters.

\begin{tabular}{|c|c|c|c|c|c|}
\hline \multirow{2}{*}{$\frac{\text { AIV-TEC-MEG-084-3.6 }}{\text { COLL-D5 }}$} & \multirow[b]{4}{*}{ Requirement } & \multirow[t]{3}{*}{ Manufacturer } & \multirow[t]{3}{*}{ с10 } & \multirow[b]{4}{*}{ Measurement } & \multirow[b]{4}{*}{ Instrument or method } \\
\hline & & & & & \\
\hline Hellma CaF2 & & & & & \\
\hline Parameter & & Measurement & Measurement & & \\
\hline Surface 1 & & Test plate & Surface 1 & Surface 2 & \\
\hline $\operatorname{RoC}(\mathrm{mm})$ & $\begin{array}{c}344.5+/-0.5 \\
\mathrm{~mm}\end{array}$ & - & 344.173 & - & WYCO Interferometer \\
\hline Used aperture $\Phi(\mathrm{mm})$ & 236 & _ & 236 & _ & \\
\hline Irr in used aperture & $3 \mathrm{fr}$ & - & 0.699 & - & $\begin{array}{l}\text { WYCO Interferometer } \\
\text { subaperture stitiching }\end{array}$ \\
\hline Irr in $\Phi=160$ & 2 fi & - & 0.462 & - & $\begin{array}{l}\text { WYCO Interferometer } \\
\text { subaperture stitiching }\end{array}$ \\
\hline MIIL 13830 SD & $60 / 40$ & - & $60 / 40$ & - & $\begin{array}{l}\text { Visual inspection vs. calibrated } \\
\text { reference }\end{array}$ \\
\hline Protective chamfer & $1.0 \times 45^{\circ}$ & _ & $1.0 \times 45^{\circ}$ & _ & Graduate reticle \\
\hline AR Coating $370-980 \mathrm{~mm}$ & no coating & - & & _ & \\
\hline Surface 2 & & Test plate & Surface 1 & Surface 2 & \\
\hline RoC (mm) & $-542.5 \pm 0.5$ & 542.81 & _ & 542.09 & WYCO Interferometer \\
\hline Used aperture $\Phi(\mathrm{mm})$ & 236 & 236 & _ & 236 & \\
\hline Irr in used aperture & $2 \mathrm{fr}$ & 0.48 & - & 1.19 & $\begin{array}{c}\text { Newton interferometer / Image } \\
\text { Analysis Software }\end{array}$ \\
\hline Irr in $\Phi=160$ & $0.5 \mathrm{fr}$ & 0.24 & - & 0.35 & $\begin{array}{c}\text { Newton interferometer / Image } \\
\text { Analysis Software }\end{array}$ \\
\hline MII 13830 SD & $40 / 20$ & - & - & $40 / 20$ & \begin{tabular}{|c|}
$\begin{array}{c}\text { Visual inspection } v s . \text { calibrated } \\
\text { reference }\end{array}$ \\
\end{tabular} \\
\hline Protective chamfer & $1.0 \times 45^{\circ}$ & - & - & $1.0 \times 45^{\circ}$ & Graduate reticle \\
\hline $\begin{array}{l}\text { AR coating } 370-980 \mathrm{~nm} \\
\mathrm{R}<1.3 \%\end{array}$ & Tav $>98.7 \%$ & - & - & 98.9 & CARY spectrometer \\
\hline \multicolumn{6}{|l|}{ Lens } \\
\hline Edge diameter (mm) & $\begin{array}{c}250+0.0 /- \\
0.015\end{array}$ & & - & 250.005 & CMM \\
\hline Central thickness* $(\mathrm{mm})$ & $45 \pm 0.15$ & - & _ & 44.933 & CMMM \\
\hline Wedge (arcmin) & 2 & _ & _ & _1.83 & Centering LOH machine \\
\hline
\end{tabular}


Table 6. Camera lens CAM-D1 as-built parameters.

\begin{tabular}{|c|c|c|c|c|c|}
\hline \multirow{2}{*}{\begin{tabular}{|c|}
$\begin{array}{c}\text { AIV-TEC-MEG-084- } \\
3.7\end{array}$ \\
CAM-Dl \\
\end{tabular}} & \multirow[b]{4}{*}{ Requirement } & \multirow[t]{3}{*}{ Manufacturer } & \multirow[t]{3}{*}{ CIO } & \multirow[b]{4}{*}{ Measurement } & \multirow[b]{4}{*}{ Instrument or method } \\
\hline & & & & & \\
\hline Hellma CaF2 & & & & & \\
\hline Parameter & & Measurement & Measurement & & \\
\hline Surface 1 & & Test plate & Surface 1 & & +2 \\
\hline $\mathrm{RoC}(\mathrm{mm})$ & $\begin{array}{c}435.9+/-0.4 \\
\mathrm{~mm}\end{array}$ & & $436.160 \mathrm{~mm}$ & & Wyko Interferometer \\
\hline Used aperture $\Phi(\mathrm{mm})$ & $216 \mathrm{~mm}$ & - & $\Phi=216 \mathrm{~mm}$ & - & $\begin{array}{l}\text { Wyko Interferometer and } \\
\text { stitching ZEEKO software }\end{array}$ \\
\hline Irr in used aperture & $1 \mathrm{fr}$ & - & $0.31664 \mathrm{fr}$ & - & $\begin{array}{l}\text { Wyko Interferometer and } \\
\text { stitching ZEEKO software }\end{array}$ \\
\hline Irr in $\Phi=160$ & $0.5 \mathrm{fr}$ & - & $0.21040 \mathrm{fr}$ & - & $\begin{array}{l}\text { Wyko Interferometer and } \\
\text { stitching ZEEKO software }\end{array}$ \\
\hline MIL 13830 SD & $40 / 20$ & _ & $40 / 20$ & $\ldots$ & Mil. Std. Norm. \\
\hline Protective chamfer & $1.0 \times 45^{\circ}$ & - & $1.0 \times 45^{\circ}$ & _ & Graduated reticle \\
\hline $\begin{array}{l}\text { AR Coating } 370-980 \\
\mathrm{~nm} \\
\mathrm{R}>1.3 \%\end{array}$ & Tav $>98.7 \%$ & & Tav $=98.7 \%$ & & CARY spectrometer \\
\hline Surface 2 & & & & Surface2 & \\
\hline $\mathrm{RoC}(\mathrm{mm})$ & $\begin{array}{c}231.7+/-0.2 \\
\mathrm{~mm}\end{array}$ & $231.796 \mathrm{~mm}$ & - & $231.758 \mathrm{~mm}$ & Wyko Interferometer \\
\hline Used aperture $\Phi(\mathrm{mm})$ & $216 \mathrm{~mm}$ & $130 \mathrm{~mm}$ & _ & $216 \mathrm{~mm}$ & Image Analysis Software \\
\hline Irr in used aperture & $2 \mathrm{fr}$ & $0.256 \mathrm{fr}$ & - & $1.03 \mathrm{fr}$ & Image Analysis Software \\
\hline Irr in $\Phi=160$ & $1 \mathrm{fr}$ & $0.256 \mathrm{fr}$ & _ & $1.03 \mathrm{fr}^{1}$ & Image Analysis Software \\
\hline MIL 13830 SD & $60 / 40$ & _ & _ & $60 / 40$ & Mil. Std. Norm. \\
\hline Protective chamfer & $1.0 \times 45^{\circ}$ & _- & _- & $1.0 \times 45^{\circ}$ & Graduated reticle \\
\hline $\begin{array}{l}\text { AR coating } 370-980 \\
\mathrm{~nm}\end{array}$ & no coating & - & - & - & \\
\hline \multicolumn{6}{|l|}{ Lens } \\
\hline Edge diameter (mm) & $\begin{array}{l}236+0.0 /- \\
0.015 \mathrm{~mm}\end{array}$ & & & $235.998 \mathrm{~mm}$ & CMM \\
\hline $\begin{array}{l}\text { Central thickness* } \\
(\mathrm{mm})\end{array}$ & $\begin{array}{c}60.0+/-0.1 \\
\mathrm{~mm}\end{array}$ & & & $59.945 \mathrm{~mm}$ & $\mathrm{CMM}$ \\
\hline Wedge (arcmin) & 2 & & & 1.8 & Centering machine \\
\hline
\end{tabular}

Proc. of SPIE Vol. 9908 990885-7 
Table 7. Camera lens CAM-D2 as-built parameters.

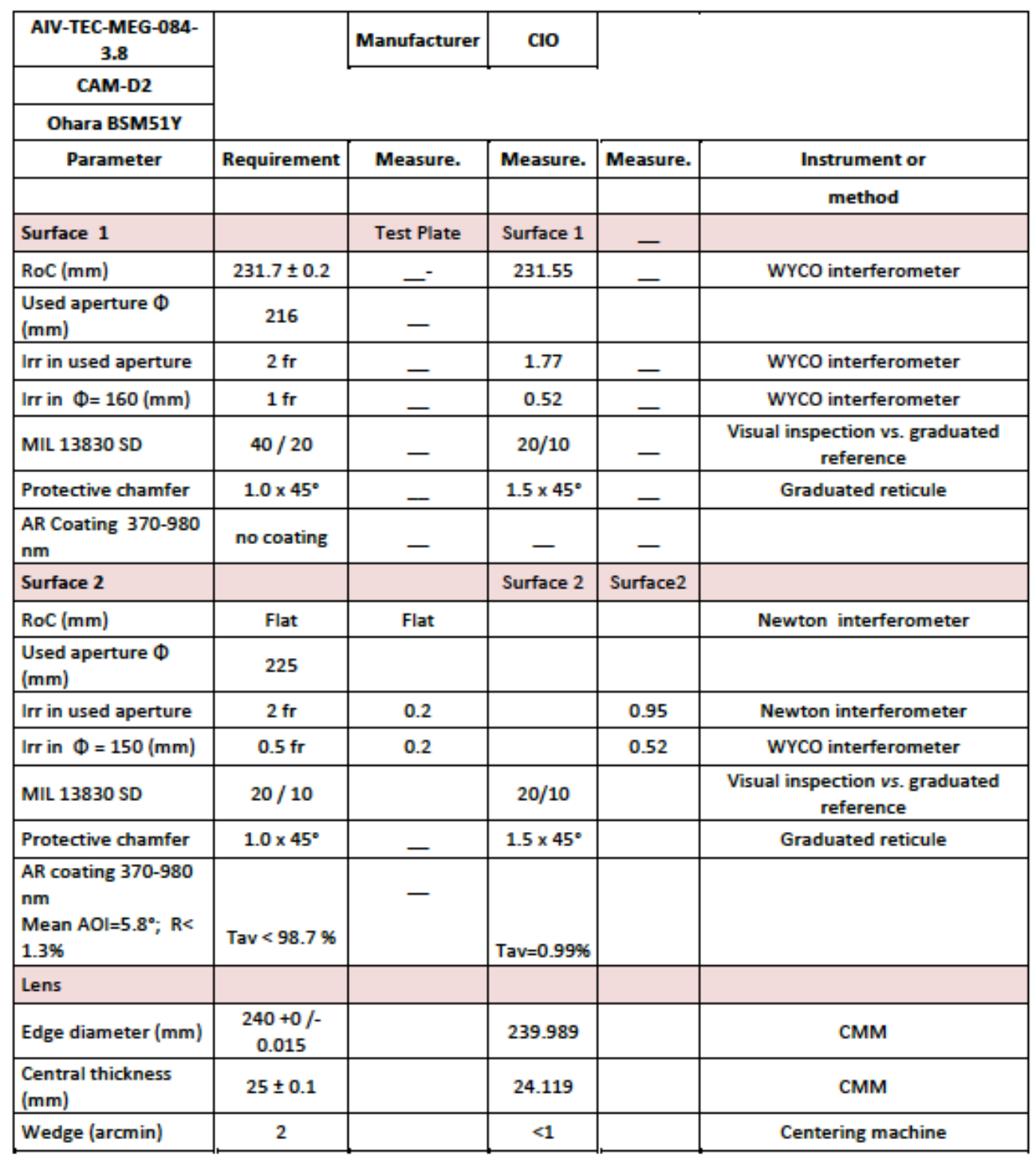


Table 8. Camera lens CAM-D3 as-built parameters.

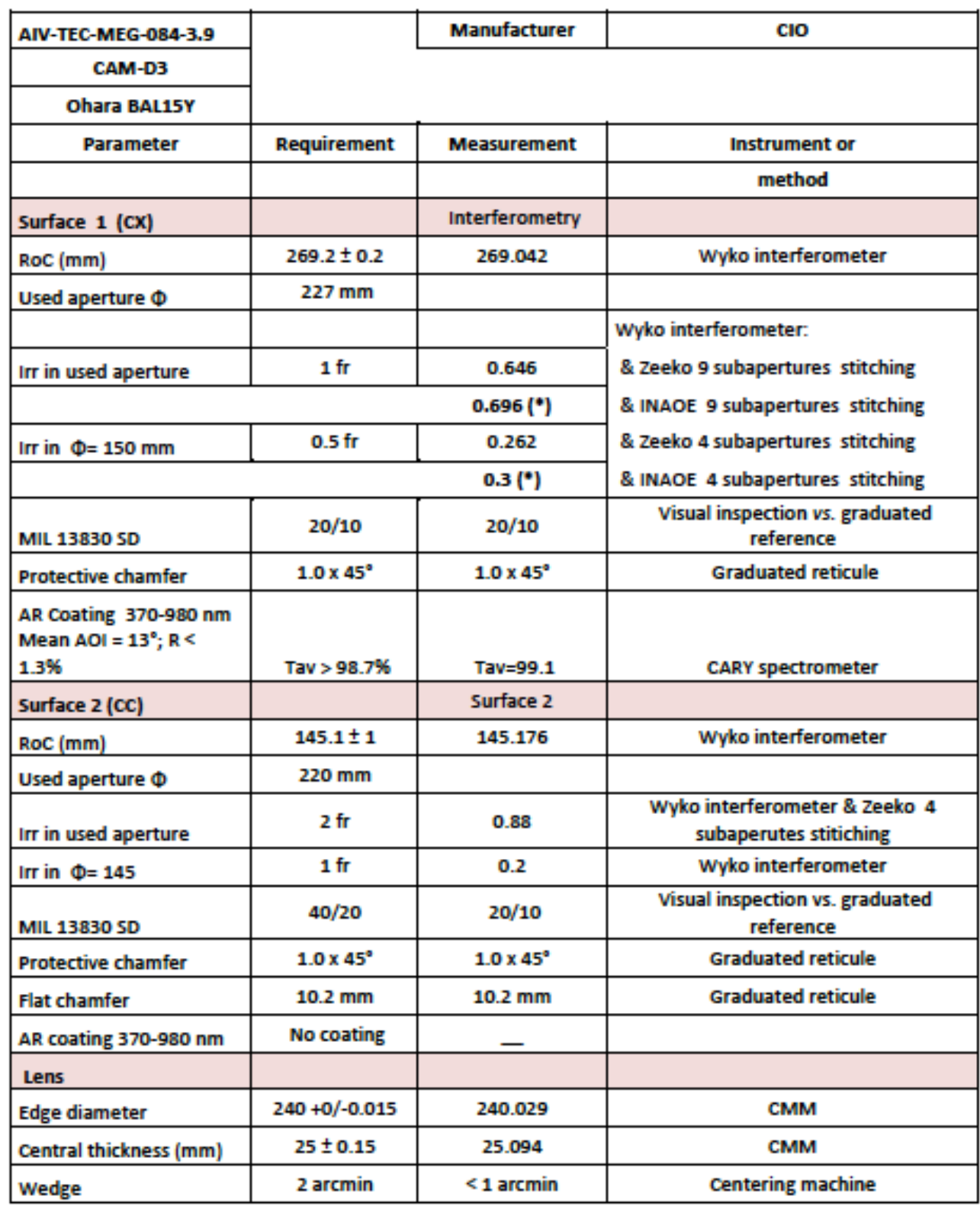

Proc. of SPIE Vol. $9908990885-9$ 
Table 9. Camera lens CAM-D4 as-built parameters.

\begin{tabular}{|c|c|c|c|c|c|}
\hline AIV-TEC-MEG-084-3.10 & & Manufacturer & CIO & & \\
\hline \multicolumn{6}{|l|}{ CAM-D4 } \\
\hline \\
\hline Parameter & Requirement & Measurement & Measurement & Measurement & Instrument or method \\
\hline Surface 1 & & Test plate & Surface 1 & Surface 2 & \\
\hline $\mathrm{RoC}(\mathrm{mm})$ & \begin{tabular}{|c|}
$145.1+/-0.1$ \\
$\mathrm{~mm}$
\end{tabular} & 145.118 & 145.064 & - & WYCO Interferometer \\
\hline Used aperture $\Phi(\mathrm{mm})$ & 215 & 100 & 215 & _ & Vecco Analysis Software \\
\hline Irr in used aperture & $2 \mathrm{fr}$ & 0.24 & 1.04 & _ & Vecco Analysis Software \\
\hline Irr in $\Phi=145$ & $1 \mathrm{fr}$ & _ & 0.70 & _ & Vecco Analysis Software \\
\hline MIL 13830 SD & $40 / 20$ & - & $40 / 20$ & - & Mil. Std. Norm. \\
\hline Protective chamfer & $1.0 \times 45^{\circ}$ & - & $1.0 \times 45^{\circ}$ & _- & Graduate reticle \\
\hline AR Coating $370-980 \mathrm{~nm}$ & no coating & _- & & _- & \\
\hline Surface 2 & & Test plate & Surface 1 & Surface 2 & \\
\hline $\mathrm{RoC}(\mathrm{mm})$ & Flat & _ & _ & Flat & \\
\hline Used aperture $\Phi(\mathrm{mm})$ & 215 & - & - & 215 & $\begin{array}{l}\text { WYCO Interferometer and } \\
\text { stitching ZEEKO software }\end{array}$ \\
\hline Irr in used aperture & $2 \mathrm{fr}$ & - & - & 1.15 & $\begin{array}{l}\text { Wyko Interferometer and } \\
\text { stitching ZEEKO software }\end{array}$ \\
\hline Irr in $\Phi=140$ & $0.5 \mathrm{fr}$ & _ & _ & 0.266 & WYCO Interferometer \\
\hline MIL 13830 SD & $40 / 20$ & _ & _ & $40 / 20$ & Mil. Std. Norm. \\
\hline Protective chamfer & $1.0 \times 45^{\circ}$ & - & - & $1.0 \times 45^{\circ}$ & Graduate reticle \\
\hline $\begin{array}{l}\text { AR coating } 370-980 \mathrm{~nm} \\
\mathrm{R}<1.3 \%\end{array}$ & Tav $>98.7 \%$ & - & - & Tav $=99 \%$ & CARY spectrometer \\
\hline \multicolumn{6}{|l|}{ Lens } \\
\hline Edge diameter $(\mathrm{mm})$ & $\begin{array}{c}220+0.0 /- \\
0.015\end{array}$ & & - & 219.995 & $\begin{array}{c}\text { Coordinate Measuring } \\
\text { Machine } \\
\end{array}$ \\
\hline Central thickness* $(\mathrm{mm})$ & $60 \pm 0.1$ & - & - & 60.046 & $\begin{array}{c}\text { Coordinate Measuring } \\
\text { Machine }\end{array}$ \\
\hline Wedge (arcmin) & 2 & _ & _ & 1'50" & Centering LOH machine \\
\hline
\end{tabular}

Proc. of SPIE Vol. $9908990885-10$ 
Table 10. Camera lens CAM-S5 as-built parameters.

\begin{tabular}{|c|c|c|c|c|c|}
\hline $\begin{array}{c}\text { AIV-TEC-MEG-084- } \\
3.10 \\
\end{array}$ & & Manufacturer & CIO & & \\
\hline \multicolumn{6}{|l|}{ CAM-S5 } \\
\hline \multicolumn{6}{|l|}{ Hellma CaF2 } \\
\hline Parameter & Requirement & Measurement & Measurement & Measurement & Instrument or method \\
\hline Surface 1 & & Test plate & Surface 1 & Surface 2 & \\
\hline $\operatorname{RoC}(\mathrm{mm})$ & $\begin{array}{c}156+/-0.1 \\
\mathrm{~mm}\end{array}$ & 156.036 & 156.084 & - & WYCO Interferometer \\
\hline Used aperture $\Phi(\mathrm{mm})$ & 215 & _ & 215 & _ & Vecco Analysis Software \\
\hline Irr in used aperture & $2 \mathrm{fr}$ & - & 0.92 & - & Vecco Analysis Software \\
\hline Irr in $\Phi=120$ & $0.5 \mathrm{fr}$ & 0.443 & $0.8097^{1}$ & - & Vecco Analysis Software \\
\hline MIL 13830 SD & $40 / 20$ & - & $40 / 20$ & - & Mil. Std. Norm. \\
\hline Protective chamfer & $1.0 \times 45^{\circ}$ & - & $1.0 \times 45^{\circ}$ & _ & Graduate reticle \\
\hline $\begin{array}{l}\text { AR Coating } 370-980 \mathrm{~nm} \\
\mathrm{R}<1.3 \% \\
\end{array}$ & Tav $>98.7 \%$ & - & Tav $=99.1 \%$ & - & \\
\hline Surface 2 & & Test plate & Surface 1 & Surface 2 & \\
\hline $\operatorname{RoC}(\mathrm{mm})$ & $\begin{array}{c}1143+/-0.8 \\
\mathrm{~mm}\end{array}$ & 1143.19 & - & 1143.78 & \\
\hline Used aperture $\Phi(\mathrm{mm})$ & 210 & 210 & - & 210 & $\begin{array}{l}\text { WYCO Interferometer and } \\
\text { stitching ZEEKO software }\end{array}$ \\
\hline Irr in used aperture & $2 \mathrm{fr}$ & 0.364 & - & 1.28 & $\begin{array}{c}\text { Wyko Interferometer and } \\
\text { stitching ZEEKO software }\end{array}$ \\
\hline Irr in $\Phi=100$ & $0.5 \mathrm{fr}$ & 0.101 & _- & 0.31 & WYCO Interferometer \\
\hline MIL 13830 SD & $40 / 20$ & _- & _- & $40 / 20$ & Mil. Std. Norm. \\
\hline Protective chamfer & $1.0 \times 45^{\circ}$ & - & - & $1.0 \times 45^{\circ}$ & Graduate reticle \\
\hline $\begin{array}{l}\text { AR coating } 370-980 \mathrm{~nm} \\
\mathrm{R}<1.3 \%\end{array}$ & Tav $>98.7 \%$ & - & Tav $=98.7 \%$ & & CARY spectrometer \\
\hline \multicolumn{6}{|l|}{ Lens } \\
\hline Edge diameter (mm) & $\begin{array}{l}220+0.0 /- \\
0.015\end{array}$ & - & - & 219.990 & $\begin{array}{c}\text { Coordinate Measuring } \\
\text { Machine } \\
\end{array}$ \\
\hline Central thickness* (mm) & $62 \pm 0.1$ & - & - & $61.30^{1}$ & $\begin{array}{c}\text { Coordinate Measuring } \\
\text { Machine } \\
\end{array}$ \\
\hline Wedge (arcmin) & 2 & - & - & 1'50" & Centering LOH machine \\
\hline
\end{tabular}


Table 11. Camera lens CAM-S6 as-built parameters.

\begin{tabular}{|c|c|c|c|c|}
\hline AIV-TEC-MEG-084-3.12 & & Manufacturer & cio & \\
\hline CAM-56 & & & & \\
\hline Ohara S-LAH55V & & & & \\
\hline \multirow[t]{2}{*}{ Parameter } & Requirement & Measurement & Measurement & Instrument or \\
\hline & & & & method \\
\hline Surface 1 & & Test plate & $\begin{array}{c}\text { Surface } \\
1 / \text { Newton }\end{array}$ & \\
\hline RoC & $176.4+/-0.2$ & 176.348 & 176.353 & $\begin{array}{c}\text { Slide optical } \\
\text { bench/wrcoInterferometer }\end{array}$ \\
\hline Used aperture $\Phi$ & 130 & & & \\
\hline Irr in used aperture & $1 \mathrm{fr}$ & 0.346 & 0.49 & Newton interferometer \\
\hline Irr in $\Phi=60$ & $0.5 \mathrm{fr}$ & 0.056 & 0.18 & Newton interferometer \\
\hline MIL 13830 SD & $20 / 10$ & - & $20 / 10$ & $\begin{array}{c}\text { Visual inspection vs. graduated } \\
\text { reference }\end{array}$ \\
\hline Protective chamfer & $1.0 \times 45^{\circ}$ & - & $1.0 \times 45^{\circ}$ & Graduate reticule \\
\hline $\begin{array}{l}\text { AR coating } 370-980 \mathrm{~nm} \\
\text { Mean } A O I=9.3^{\circ} ; R< \\
1.3 \%\end{array}$ & Tav $>98.7 \%$ & 一 & Tav $=98.76 \%$ & CARY spectrometer \\
\hline Surface 2 & & & Surface 2 & \\
\hline Roc & $365.8+/-0.3$ & & 366.023 & $\begin{array}{l}\text { Slide optical bench/ WYco } \\
\text { Interferometer }\end{array}$ \\
\hline Used aperture $\Phi$ & 102 & & & \\
\hline Irr in used aperture & $1 \mathrm{fr}$ & - & $0.139 \mathrm{fr}$ & WYco interferometer \\
\hline Irr in $\Phi=50$ & 0.5 & - & $0.021 \mathrm{fr}$ & WYco interferometer \\
\hline MIL 13830 SD & $20 / 10$ & 一 & $20 / 10$ & $\begin{array}{l}\text { Visual inspection vs. graduated } \\
\text { reference } \\
\end{array}$ \\
\hline Protective chamfer & $1.0 \times 45^{\circ}$ & - & $1.0 \times 45^{\circ}$ & Graduate reticule \\
\hline Flat chamfer (mm) & 15 & - & 15 & Graduate reticule \\
\hline $\begin{array}{l}\text { AR coating } 370-980 \mathrm{~nm} \\
\text { Mean } A O I=11.7^{\circ} ; R< \\
1.3 \%\end{array}$ & Tav $>98.7 \%$ & - & Tav $=98.71 \%$ & CARY spectrometer \\
\hline \multicolumn{5}{|l|}{ Lens } \\
\hline Edge diameter & $140+0 /-0.015$ & - & 139.994 & CMM \\
\hline Central thickness & $40.0+/-0.1$ & - & 40.01 & CMM \\
\hline Wedge (arcmin) & 2 & - & 1.5 & Centering machine \\
\hline
\end{tabular}

Proc. of SPIE Vol. 9908 990885-12

Downloaded From: http://proceedings.spiedigitallibrary.org/ on 03/08/2017 Terms of Use: http://spiedigitallibrary.org/ss/termsofuse.aspx 
Table 12. Camera lens CAM-S7 as-built parameters.

\begin{tabular}{|c|c|c|c|}
\hline AIV-TEC-MEG-084-3.13 & & Manufacturer & INAOE \\
\hline \multicolumn{4}{|l|}{ CAM-S7 } \\
\hline Ohara S-NBH8 & & & \\
\hline \multirow[t]{2}{*}{ Parameter } & Requirement & Measurement & Instrument or \\
\hline & & & method \\
\hline Surface 1 & & Surface 1 & \\
\hline $\operatorname{RoC}(\mathrm{mm})$ & $-162.5+/-0.2$ & $-162.750^{1}$ & Nodal slide optical bench \\
\hline Used aperture $\Phi(\mathrm{mm})$ & 97 & & \\
\hline Irr (P-V) in used aperture & $2 \mathrm{fr}$ & 0.3882 & ZYGO Interferometer \\
\hline $\operatorname{Irr}(P-V)$ in $\Phi=20 \mathrm{~mm}$ & $0.25 \mathrm{fr}$ & 0.0533 & ZYGO Interferometer \\
\hline MIL $13830 \mathrm{SD}$ & $20 / 10$ & $40 / 10^{1}$ & Visual inspection vs. calibrated reference \\
\hline Protective chamfer & $1.0 \times 45^{\circ} \max$ & $1.0 \times 45^{\circ} \max$ & Graduated reticle \\
\hline $\begin{array}{l}\text { AR Coating } 370-980 \mathrm{~nm} \\
\text { AOI }=20.3^{\circ} ; \mathrm{R}>1.3 \%\end{array}$ & Tav $>98.7 \%$ & $T=99.5 \%$ & CARY spectrometer \\
\hline Surface 2 & & Surface 2 & \\
\hline RoC (mm) & $219.5+/-0.2$ & $219.85^{1}$ & Nodal slide optical bench \\
\hline Used aperture $\Phi(\mathrm{mm})$ & 86 & & \\
\hline $\operatorname{Irr}(\mathrm{P}-\mathrm{V})$ in used aperture & $2 \mathrm{fr}$ & 0.1153 & ZYGO Interferometer \\
\hline $\operatorname{Irr}(P-V)$ in $\Phi=10 \mathrm{~mm}$ & $0.25 \mathrm{fr}$ & 0.1172 & ZYGO Interferometer \\
\hline MIL 13830 SD & $20 / 10$ & $20 / 10$ & Visual inspection vs. calibrated reference \\
\hline Protective chamfer & $1.0 \times 45^{\circ} \max$ & $1.0 \times 45^{\circ} \max$ & Graduated reticle \\
\hline $\begin{array}{l}\text { AR coating } 370-980 \mathrm{~nm} \\
\text { AOI }=14.8^{\circ} ; R>1.3 \%\end{array}$ & Tav $>98.7 \%$ & $\mathrm{~T}=99.3 \%$ & CARY spectrometer \\
\hline \multicolumn{4}{|l|}{ Lens } \\
\hline Edge diameter $(\mathrm{mm})$ & $110-0.02 /-0.08$ & 109.95 & Mitutoyo Calibrator \pm 0.010 \\
\hline Central thickness* $(\mathrm{mm})$ & $30+/-0.1$ & 30.05 & Mitutoyo Calibrator \pm 0.010 \\
\hline Wedge & 2 arcmin & 0.06 & Mitutoyo Calibrator \pm 0.010 \\
\hline
\end{tabular}

Proc. of SPIE Vol. 9908 990885-13 
Table 13. Field lens as-built parameters.

\begin{tabular}{|c|c|c|c|c|c|}
\hline AIV-TEC-MEG-084-3.1 & & Manufacturer & INAOE & & \\
\hline \multicolumn{6}{|l|}{ Field lens } \\
\hline \\
\hline Parameter & Requirement & Measurement & Measurement & Measurement & Instrument or method \\
\hline Surface 1 & & Test plate & Surface 1 & Surface 2 & \\
\hline $\mathrm{RoC}(\mathrm{mm})$ & $-2147.6 \pm 2$ & _- & -2146.7 & _- & Oriel optics slide bench \pm 1 \\
\hline Used aperture $\Phi(\mathrm{mm})$ & 260 & - & & - & \\
\hline Irr in used aperture & $4 \mathrm{fr}$ & _ & $1.043 \mathrm{fr}$ & _ & ZYGO Interferometer \\
\hline Irr in $\Phi=10$ & $0.25 \mathrm{fr}$ & _ & $0.0232 \mathrm{fr}$ & _ & ZYGO Interferometer \\
\hline MIL 13830 SD & $20 / 10$ & - & $10 / 5$ & - & $\begin{array}{l}\text { Visual inspection vs. calibrated } \\
\text { reference (Brysen optical corp. } \\
7641866 \text { ) }\end{array}$ \\
\hline Protective chamfer & $1.0 \times 45^{\circ}$ & _ & $1.3 \times 45^{\circ}$ & _ & Mitutoyo cal. \pm 0.010 \\
\hline \multicolumn{6}{|l|}{ AR Coating $370-980 \mathrm{~nm}$} \\
\hline $\mathrm{R}<1.3 \%$ & Tav $>98.7 \%$ & & Tav $=99.21 \%$ & & CARY spectrometer \\
\hline Surface 2 & & Test plate & Surface 1 & Surface 2 & \\
\hline $\mathrm{RoC}(\mathrm{mm})$ & $-1731.8 \pm 2$ & -1731.89 & _ & & Oriel optics slide bench \pm 1 \\
\hline Used aperture $\Phi(\mathrm{mm})$ & 260 & 288 & _ & & \\
\hline Irr in used aperture & $4 \mathrm{fr}$ & $0.4844 \mathrm{fr}$ & - & $1.312 \mathrm{fr}$ & Newton interferometer \\
\hline Irr in $\Phi=10$ & $0.25 \mathrm{fr}$ & $0.0508 \mathrm{fr}$ & _ & $0.0993 \mathrm{fr}$ & Newton interferometer \\
\hline MIL 13830 SD & $20 / 10$ & $20 / 10$ & - & $10 / 5$ & $\begin{array}{l}\text { Visual inspection vs. calibrated } \\
\text { reference (Brysen optical corp. } \\
7641866 \text { ) }\end{array}$ \\
\hline Protective chamfer & $1.0 \times 45^{\circ}$ & _ & _ & $1.3 \times 45^{\circ}$ & Mitutoyo cal. \pm 0.010 \\
\hline $\begin{array}{l}\text { AR coating } 370-980 \mathrm{~nm} \\
\mathrm{R}<1.3 \%\end{array}$ & Tav $>98.7 \%$ & - & - & Tav $=99.19 \%$ & CARY spectrometer \\
\hline \multicolumn{6}{|l|}{ Lens } \\
\hline Edge diameter (mm) & $\begin{array}{c}274+0 /- \\
0.015\end{array}$ & & $273.93^{1}$ & & Mitutoyo cal. \pm 0.010 \\
\hline Central thickness* $(\mathrm{mm})$ & $30 \pm 0.1$ & & $30.17^{1}$ & & Mitutoyo cal. \pm 0.010 \\
\hline Wedge (arcmin) & 2 & & 0.03 & & Mitutoyo cal. \pm 0.010 \\
\hline
\end{tabular}



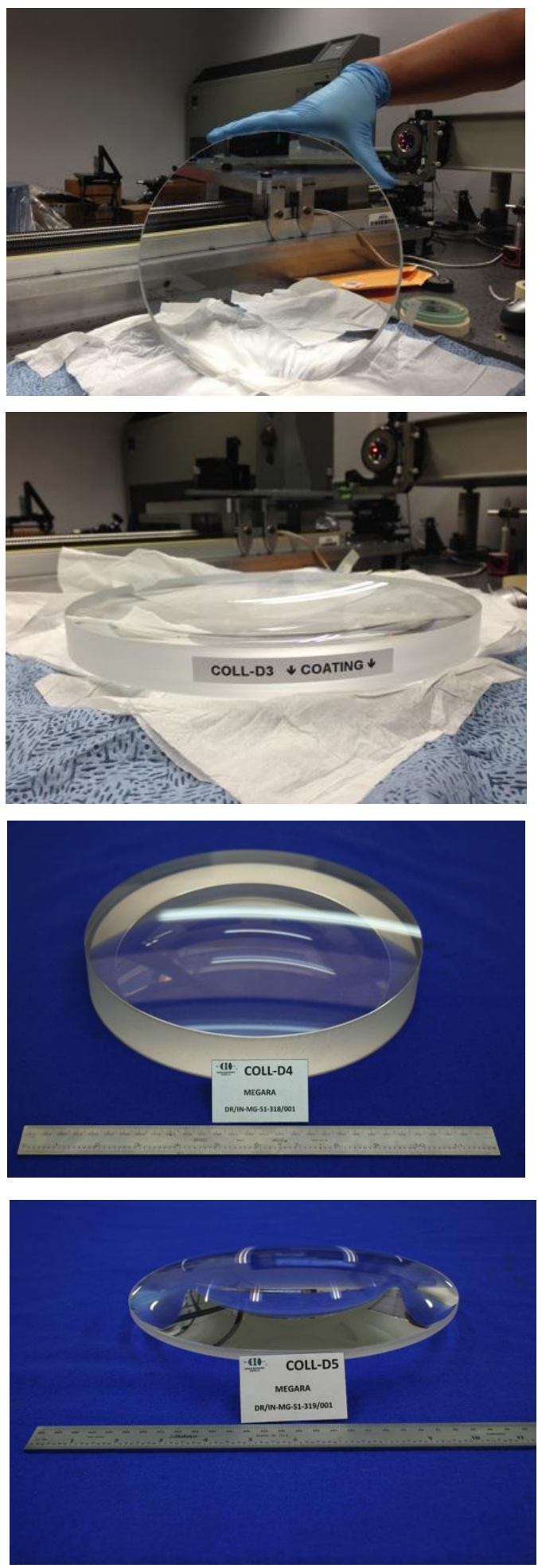

Figure 1. Collimator lenses. From top to bottom: COLL-D2 (PMB2Y), COLL-D3 (BSM51Y), COLL-D4 (PBM8Y) and COLL-D5 $(\mathrm{CaF} 2)$. 

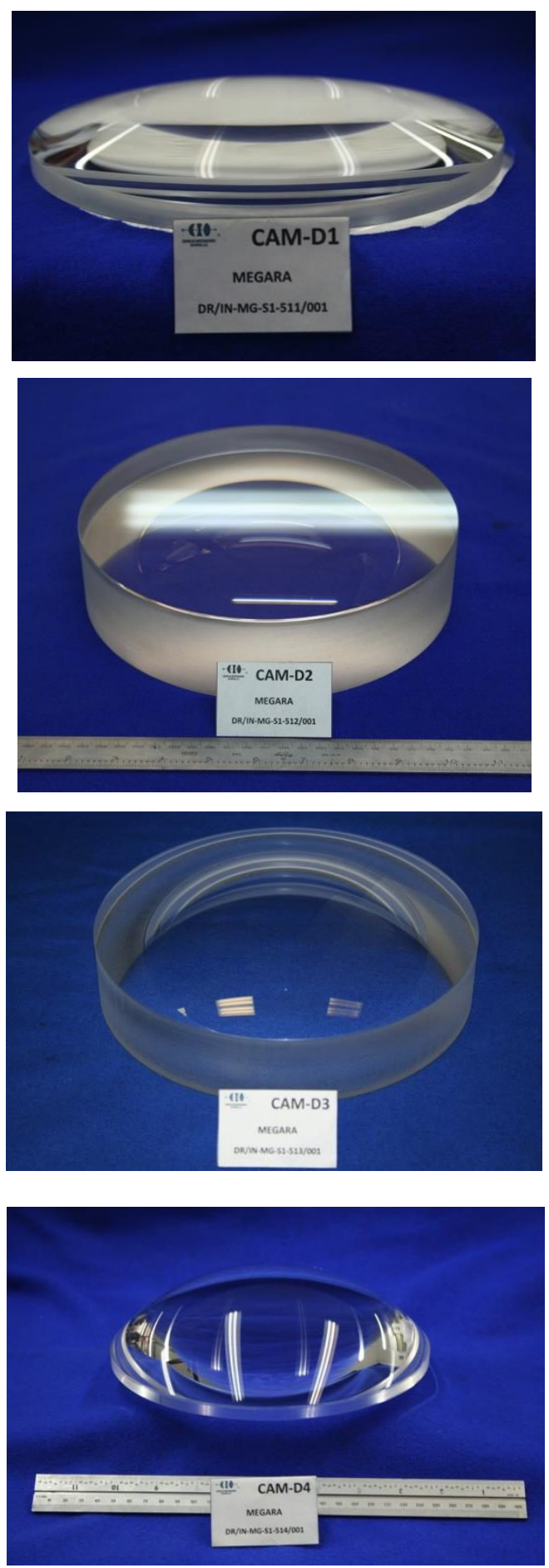

Figure 2. Camera lenses. From top to bottom: CAM-D1 (CaF2), CAM-D2 (BSM51Y), CAM-D3 (BAL15Y) and CAM-D4 (CaF2).

Proc. of SPIE Vol. $9908990885-16$ 

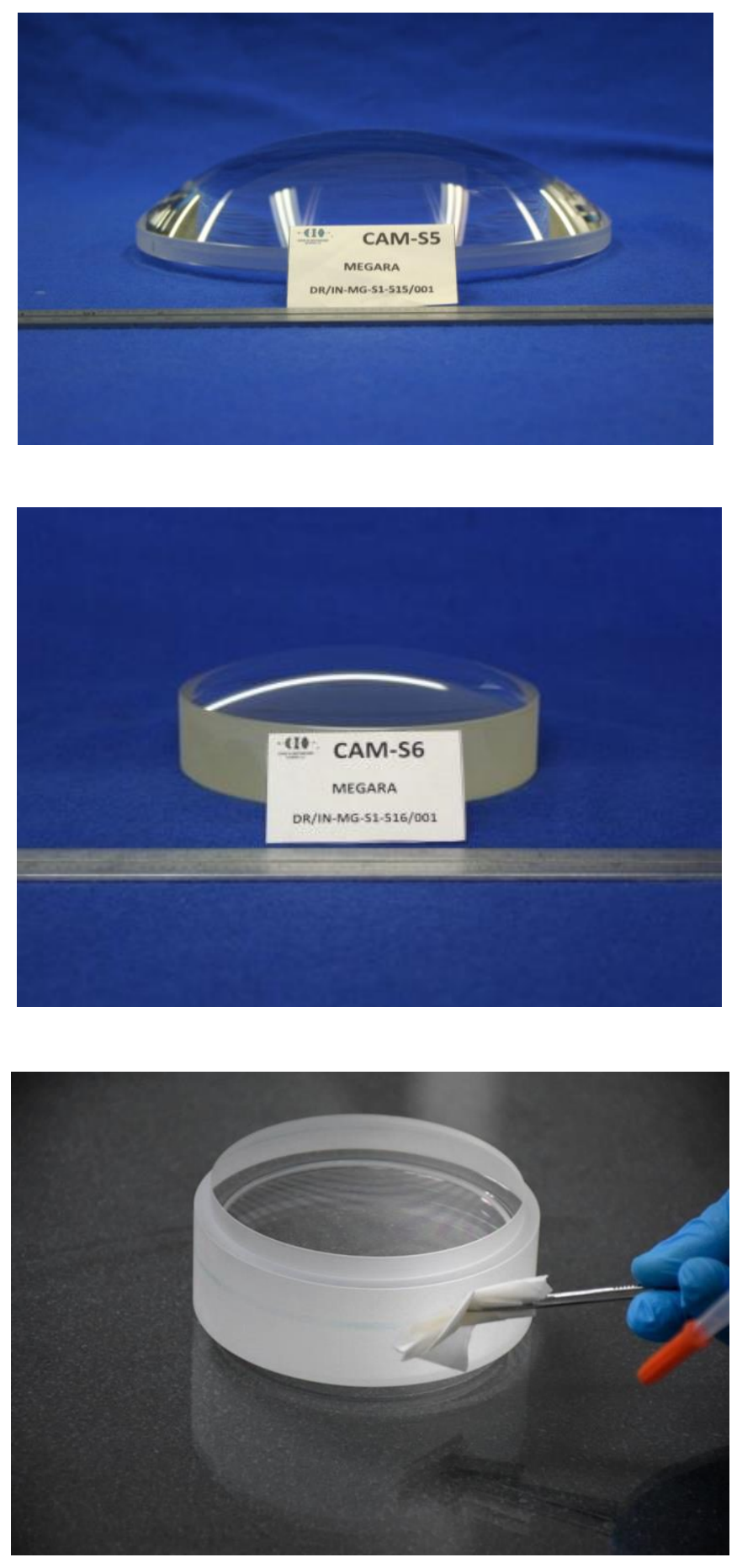

Figure 3. Camera lenses. From top to bottom: CAM-S5 (CaF2), CAM-S6 (S-LAH55) and CAM-S7 (S-NBH8).

Proc. of SPIE Vol. $9908990885-17$ 


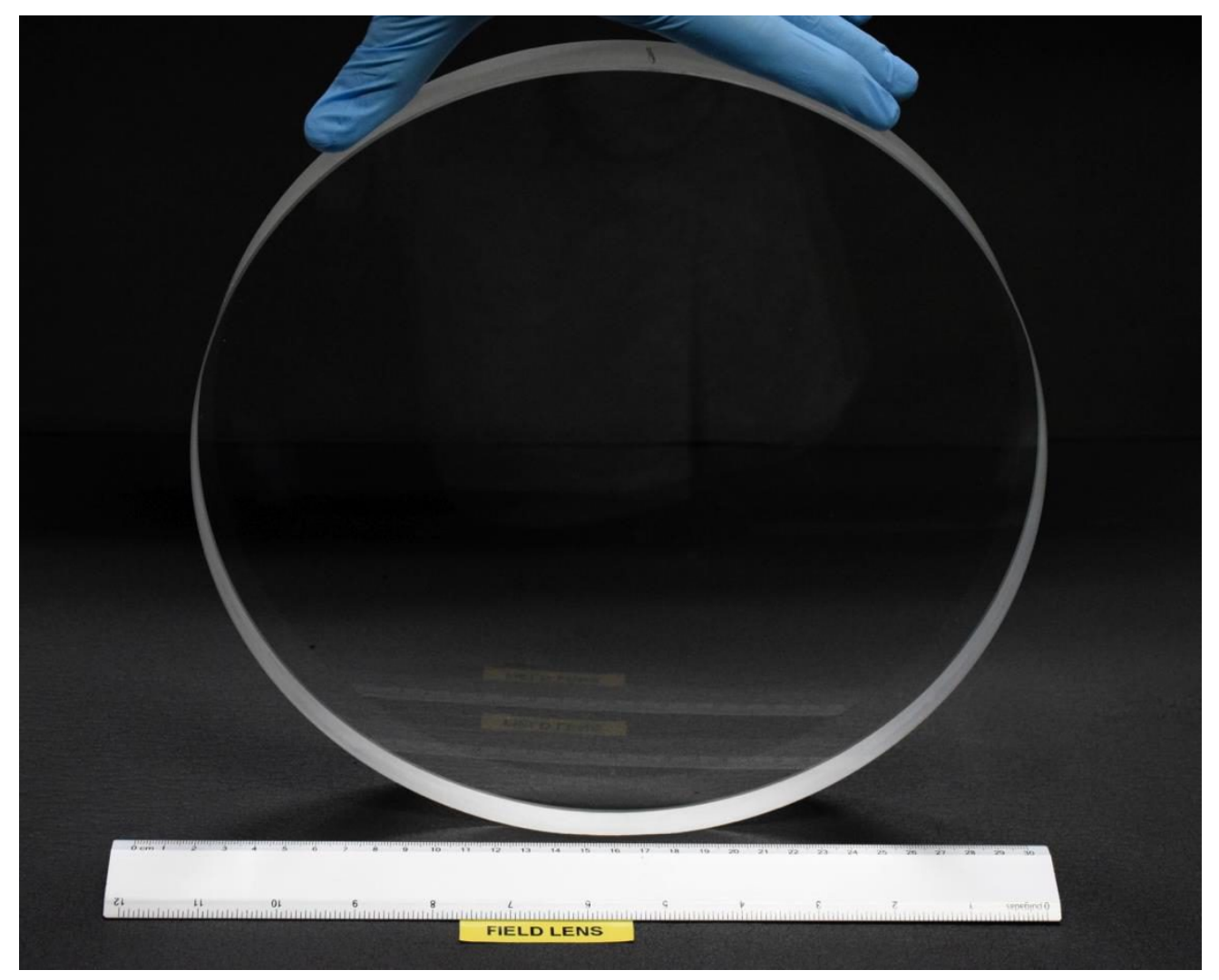

Figure 4. Field lens. Ohara SK1300 (fused Silica). 274 mm diameter.

\section{CONCLUSIONS}

MEGARA collimator and camera lenses and the field lens - manufactured at INAOE and CIO -except for the asphericcomply the specifications. There are 9 different materials with individual complexity regarding polishing and testing. From Ohara, SK1300, PBM2Y, BSM51Y, PBM8Y, BSM51Y, BALY15Y, S-LAH55V, S-NBH8 and Hellma CaF2. The largest diameter is $270 \mathrm{~mm}$ while the smallest is $110 \mathrm{~mm}$. The doublets were cemente at INAOE. The individual elementsdoublets and singlets- were mounted in their corresponding cells and integrated in the barrels. The collimator and camera were tested at a subsystem level. Both fullfil the requeriments. MEGARA is in the acceptance, integration and verification process at Universidad Complutense de Madrid. The laboratory acceptance is schedule for September 2016 and the delivery to the observatory for December 2016.

\section{REFERENCES}

[1] Gil de Paz, A., et al. "MEGARA, the new intermediate-resolution optical IFU and MOS for GTC: getting ready for the telescope". Proc. SPIE, this volume. (2016).

[2] Ferrusca, D., et al., "Integration and characterization of the cryogenic system of MEGARA", Proc. of SPIE, this volume (2016). 
[3] Iglesias-Páramo, J., et al., "The MEGARA fiber MOS positioning tool", Proc. of SPIE, this volume (2016).

[4] Gómez-Álvarez, P., et al., "MEGARA fiber MOS assignment tool (FMAT)", Proc. of SPIE, this volume (2016).

[5] Castillo-Morales, A., et al., "MEGARA exposure time calculator", Proc. of SPIE, this volume (2016).

[6] Lefort, B., et al., "MEGARA control system", Proc. of SPIE, this volume (2016).

[7] Maldonado, M., et al., "MEGARA spectrograph mechanics and opto-mechanics in the AIV phase", Proc. of SPIE, this volume (2016).

[8] Izazaga-Pérez, R., et al., "Polishing techniques for MEGARA optics", Proc. of SPIE, volume 9912 (2016)

[9] Martínez-Delgado, I., et al., "The large MEGARA pupil elements: assembly, tests, and performance", Proc. of SPIE, this volume (2016)

[10] Pérez-Calpena, A., et al., "MEGARA: high-precision alignment system for gluing fibers and microlenses", Proc. of SPIE, this volume (2016).

[11] Ortiz, R., et al., "Design and testing of AR coatings for MEGARA optics", Proc. of SPIE, this volume (2016). 